\title{
Effectiveness of Teicoplanin Versus $\beta$-lactam for Bacteremia Due to Methicillin-Susceptible Staphylococcus Aureus: A Propensity Score-Based Study
}

Chen-Hsiang Lee ( $\square$ lee900@cgmh.org.tw)

Chang Gung Memorial Hospital

Ching-Yen Tsai

Chang Gung Memorial Hospital

I-Ling Chen

Chang Gung Memorial Hospital

Research article

Keywords: Staphylococcus aureus, $\beta$-lactam, teicoplanin, clinical outcome

Posted Date: September 8th, 2020

DOI: https://doi.org/10.21203/rs.3.rs-59403/v1

License: (c) (i) This work is licensed under a Creative Commons Attribution 4.0 International License.

Read Full License 


\section{Abstract}

Background: Many studies have shown that vancomycin is inferior to $\beta$-lactam antibiotics in terms of effectiveness in the treatment of methicillin-susceptible Staphylococcus aureus (MSSA) bacteremia. However, limited data are available regarding the comparison of clinical outcomes between patients receiving initial teicoplanin and those receiving $\beta$-lactam antibiotics for MSSA bacteremia.

Methods: Eighty-four adults with MSSA bacteremia were included: initial teicoplanin treatment group $(n=28)$ and $\beta$-lactam treatment group $(n=56)$. The two groups were further stratified based on propensity score matching according to the outcome analysis using a logistic regression model. We investigated the clinical outcomes between the groups before and after propensity score matching after treatment completion.

Results: Pittsburgh bacteremia score $\geq 4$ (odds ratio, 60.6; 95\%Cl, 7.4-496.8) was an independent risk factor for unfavorable outcome. After propensity score matching, the initial teicoplanin treatment group and the $\beta$-lactam treatment group consisted of 28 patients each. No statistically significant differences were observed in the proportions of patients with favorable outcomes and 30-day overall mortality rates between the groups before and after propensity score matching after the completion of teicoplanin or $\beta$ lactam treatment. The Kaplan-Meier 30-day survival curve also showed no significant difference between the patients receiving initial teicoplanin treatment and those receiving $\beta$-lactam treatment before and after matching (hazard ratio, $1.84,95 \% \mathrm{Cl}, 0.60-5.64$; and 3.12, 95\% $\mathrm{Cl}, 0.98-9.99$, respectively).

Conclusions: There were no significant difference in clinical outcomes between initial teicoplanin treatment and $\beta$-lactam treatment among patients with MSSA bacteremia. Pittsburgh bacteremia score $\geq 4$ was a significant risk factor for mortality.

\section{Background}

Staphylococcus aureus is one of the leading pathogens causing community-acquired and hospitalacquired bacteremia. Absence of appropriate antimicrobial treatment in patients with $S$. aureus bacteremia has a major impact on the outcomes [1]. The timing of antibiotic administration was recognized to be a major determinant of the outcome of bacteremia [2-4]. Initial empirical therapy for $S$. aureus infection may include a $\beta$-lactam or a glycopeptide such as vancomycin or teicoplanin, which is adjusted after the susceptibility test results are available $[5,6]$. Lodise et al reported an increase in mortality when treatment of nosocomial $S$. aureus bacteremia was delayed [6], and delayed initiation of appropriate therapy was common for patients with methicillin-resistant $S$. aureus (MRSA) infection [6].

Currently, it is clear that glycopeptides are not equivalent to $\beta$-lactams for the initial treatment of methicillin-susceptible $S$. aureus (MSSA) bacteremia. Many studies have indicated that vancomycin is an inferior treatment choice for MSSA strains $[7,8]$. Initial vancomycin treatment is associated with a higher incidence of delayed clearance ( $\geq 3$ days) of MSSA bacteremia [8]. In a multicenter prospective observational study, Chang et al reported that nafcillin was superior to vancomycin in preventing 
bacteriological failure (persistent bacteremia or relapse) in patients with MSSA bacteremia [9]. Another prospective study focusing on hemodialysis-dependent patients with MSSA bacteremia. Patients who were treated with vancomycin were at a higher risk of treatment failure than those who received cefazolin [10]. Treatment with vancomycin was further shown to be associated with higher mortality in patients with bacteremic pneumonia caused by MSSA [11]. Initial vancomycin therapy with subsequent deescalation to a $\beta$-lactam antibiotic may lead to worse outcomes when compared with initial $\beta$-lactam therapy for MSSA-related infectious endocarditis in intravenous drug users [12, 13].

While vancomycin and teicoplanin are used to treat gram-positive infections, there are differences in their structure, half-life, and efficacy. Teicoplanin has been widely reported to be comparable to vancomycin in efficacy but has fewer adverse effects than vancomycin [14]. Despite being structurally related to vancomycin, teicoplanin has a prolonged elimination half-life of approximately 60 hours [15]. In the Cochrane database system review, both teicoplanin and vancomycin were similarly effective in treating patients with proven or suspected infections, but the incidence of adverse effects including nephrotoxicity was lower with teicoplanin [16]. However, another meta-analysis of several trials that used adequate allocation concealment, the clinical outcome favored teicoplanin (relative risk, 0.82; 95\% confidence interval [CI], 0.63-1.06) [14]. In one of the comparative studies involving febrile neutropenic patients undergoing hematopoietic stem-cell transplantation, time to attain an effective trough concentration was shorter and the rate of clinical failure was lower with teicoplanin than vancomycin [17]. Given the scarce information on the comparison between teicoplanin and $\beta$-lactams in the treatment of MSSA infections, it remains unclear whether teicoplanin is less effectiveness than $\beta$-lactam antibiotics, as observed for vancomycin in the treatment of MSSA bacteremia. This retrospective study aimed to examine whether the outcomes between patients with MSSA bacteremia who initially received teicoplanin and those who received initial $\beta$-lactam treatment.

\section{Methods}

\section{Study design}

This retrospective study included adult patients (aged $\geq 18$ years) with MSSA bacteremia who were hospitalized at a 2,700-bed tertiary care hospital in southern Taiwan between 2012 and 2014. The study period and sample analysis were based on patient groups in our previous study $[18,19]$. Patients were included if they received either teicoplanin or a $\beta$-lactam antibiotic within 48 hours after the onset of infection and the duration of treatment with teicoplanin or $\beta$-lactam antibiotics was $\geq 72$ hours. If the patients had more than one episode of MSSA bacteremia, only the first episode was included. We collected clinical data using a standardized case report form. Adequate dosage was defined as dosage based on the manufacturer's instructions (Sanofi-Aventis, Taiwan). Teicoplanin was prescribed at a loading dose of $6 \mathrm{mg} / \mathrm{kg}$ (three loading doses 12 hours apart) followed by a maintenance dose of $6 \mathrm{mg} / \mathrm{kg}$ every 12 hours or adjusted equivalent doses for patients with impaired renal function [20, 21]. Adequate $\beta$-lactam therapy was defined as treatment with adequate dosage of oxacillin, cephalosporins, or carbapenems according to patients' renal function [21]. The antimicrobial regimen was selected at the 
discretion of the treating clinicians. All teicoplanin and $\beta$-lactam prescriptions were approved by infectious disease specialists for their indications and dosages through the antimicrobial stewardship system $[22,23]$. Patients included in the study were classified into two groups (initial teicoplanin treatment group and $\beta$-lactam treatment group) based on initial empirical antibiotic selection for MSSA bacteremia. The physicians' decision regarding de-escalating the empirical antibiotic to definite antibiotic was based on the results of drug susceptibility testing. We further stratified the groups based on propensity score matching according to the outcome analysis using a logistic regression model. The study was approved by the Institutional Review Board of Chang Gung Memorial Hospital (No. 201601482B0).

\section{Microbiological methods}

Each blood culture set (BACTEC Plus Aerobic/F and BACTEC Plus Anaerobic/F) was processed according to the BACTEC system (Becton Dickinson, Franklin Lakes, NJ, USA). MSSA was defined as an isolate of $S$. aureus that was susceptible to oxacillin (using a cefoxitin disk), as determined by the disk diffusion method according to the Clinical and Laboratory Standards Institute recommendations [24]. The minimum inhibitory concentrations (MICs) of teicoplanin were determined using Etest ${ }^{\circledR}$ teicoplanin strips (AB Biodisk, Solna, Sweden).

\section{Definitions}

The clinical severity at the time of blood sampling for cultures was stratified using the modified Pittsburgh bacteremia score. Patients with a Pittsburgh bacteremia score $\geq 4$ points were considered to be in critical illness condition [25]. Sources of bacteremia were defined according to microbiology, imaging findings, and physicians' judgment. Catheter-related infection was identified if the inserted catheter was in place for $\geq 72$ hours and the culture of the clipped distal 5-cm tip of the removed catheter yielded $\geq 15$ colonies of MSSA after rolling it on the culture medium or if the culture of purulent discharge from the catheter exit site yielded MSSA [26]. Bone and joint infection was defined based on clinical manifestations with consistent histopathological and/or radiographic findings [27]. Infective endocarditis was identified if consistent histopathological findings were observed in the valvular specimens obtained during surgery or if valvular vegetations were observed on echocardiography in patients with MSSA bacteremia [28]. Soft tissue infection was identified if the specimen sampled from the infected site yielded MSSA after culture [27]. Intra-abdominal infection was identified when cultures of the peritoneal fluid, bile, or intra-abdominal abscess grew MSSA and the infection extended into the peritoneal space with abscess formation [29]. Urinary tract infection was identified when MSSA was the only identified pathogen with $\geq 10^{5}$ colony-forming units per milliliter in urine culture [27]. Pneumonia was identified when clinical symptoms or signs of lower respiratory tract infection were accompanied by consistent radiographic findings [27]. Primary bacteremia was defined as growth of MSSA on blood culture in patients with no apparent focus of infection other than the blood culture. Adequate source control was defined as timely percutaneous or surgical intervention to drain the infected fluid, to debride the infected tissues, to drain the intra-abdominal sources of infection, or to remove the central venous catheter due to catheter-related bacteremia. 
Patients were assessed on day 7 after $\beta$-lactam or teicoplanin treatment to define early clinical response. The final clinical response was evaluated upon completion of the entire therapy. All patients were evaluated for the presence of septic shock, persistent bacteremia, fever, or leukocytosis. Favorable and unfavorable early clinical responses were based on the absence or presence of these findings, respectively [30]. The primary endpoint in this study was favorable outcome, which was defined as resolution of clinical signs and symptoms and a negative culture report at the end of therapy. Unfavorable outcomes were defined as clinical progression of sepsis, mortality, and/or blood cultures positive for MSSA at the end of teicoplanin or $\beta$-lactam treatment. The 30-day overall mortality was defined as all-cause mortality occurring within 30 days of hospitalization after the onset of MSSA bacteremia.

\section{Statistical analysis}

Continuous variables were compared using Student's t-test or Mann-Whitney U test. Dichotomous variables were compared using chi-squared test or Fisher's exact test. A $p$-value less than 0.1 was incorporated into a logistic regression model to determine independent variables associated with favorable outcome at the end of teicoplanin or $\beta$-lactam treatment. Hosmer-Lemeshow goodness-of-fit test was used to evaluate the predictive performance of the logistic regression model. The propensity score matching based on 1:1 ratio was calculated using independent predictors of favorable outcome at the end of initial teicoplanin or $\beta$-lactam treatment, which were assessed using a multivariable logistic regression model. The 30-day survival was evaluated using Kaplan-Meier curves and log-rank test. All statistical analyses were performed using IBM SPSS Statistics version 21 (IBM Corp., Armonk, NY, USA). All tests were two-tailed and $p$-values $<0.05$ were considered statistically significant.

\section{Results}

Among the 91 patients with MSSA bacteremia identified over the 3-year study period, two patients who did not receive antibiotic treatment, two patients who received initial vancomycin treatment, and three patients who were aged $<18$ years were excluded. Among the 84 adult patients included in the study, 28 (33.3\%) received initial teicoplanin treatment and 56 (66.7\%) initial $\beta$-lactam treatment. Subsequently, we stratified these two groups based on propensity score matching according to the outcome analysis using the logistic regression model (Fig. 1).

Comparisons of the demographics, comorbidities, severity of blood stream infection at onset, source of bacteremia, rate of adequate infection source control, infection by strains with teicoplanin MICs $\geq$ $1.5 \mathrm{mg} / \mathrm{L}$, and clinical outcomes between the two groups before and after propensity score matching are presented in Table 1. Before propensity score matching, no statistically significant differences were observed in terms of sex, age, length of hospital stay, and the proportion of $S$, aureus strains with teicoplanin MICs $\geq 1.5 \mathrm{mg} / \mathrm{L}$ between the groups. A significantly higher prevalence of coronary artery disease $(42.9 \%$ versus $8.9 \%, p<0.01)$ and congestive heart failure $(39.3 \%$ versus $7.1 \%, p<0.01)$ was observed in the initial teicoplanin treatment group. There was no statistically significant difference in disease severity (Pittsburgh bacteremia score $\geq 4$ ) between the groups or infection sources of 
bacteremia. The adequate infection source control rate was higher in the initial teicoplanin treatment group than in the $\beta$-lactam treatment group $(78.9 \%$ versus $41.7 \%, p=0.01)$. We did not find statistically significant differences in short-term favorable outcome, favorable outcome at the time of completion of teicoplanin or $\beta$-lactam therapy, and the 30-day overall mortality rate between the two groups (Table 1). 
Table 1

Comparisons of demographic and clinical features of patients with methicillin-susceptible Staphylococcus aureus (MSSA) bacteremia who received initial teicoplanin treatment $(n=28)$ and those who received ß-lactam antibiotic treatment $(n=56)$

\begin{tabular}{|c|c|c|c|c|c|c|}
\hline Variables & $\begin{array}{l}\text { Initial } \\
\text { teicoplanin } \\
\text { treatment } \\
\mathrm{n}=\mathbf{2 8}\end{array}$ & $\begin{array}{l}\text { Initial B- } \\
\text { lactam } \\
\text { treatment } \\
\mathrm{n}=56\end{array}$ & $P$ & $\begin{array}{l}\text { Initial } \\
\text { teicoplanin } \\
\text { treatment } \\
\mathrm{n}=\mathbf{2 8}\end{array}$ & $\begin{array}{l}\text { Initial B- } \\
\text { lactam } \\
\text { treatment } \\
\mathbf{n = 2 8}\end{array}$ & $P^{*}$ \\
\hline \multicolumn{7}{|l|}{ Demographics } \\
\hline Male gender, n (\%) & $17(60.7)$ & $37(66.1)$ & 0.64 & $17(60.7)$ & $17(60.7)$ & $\begin{array}{l}> \\
0.99\end{array}$ \\
\hline $\begin{array}{l}\text { Age, mean (range), } \\
\text { years }\end{array}$ & $75(62-81)$ & $67(55-76)$ & 0.06 & $75(62-81)$ & $75(65-84)$ & NA \\
\hline $\begin{array}{l}\text { Length of hospital } \\
\text { stay, mean (range), } \\
\text { days }\end{array}$ & $26(15-30)$ & $21(12-31)$ & 0.29 & $26(15-30)$ & $16(9-33)$ & 0.31 \\
\hline $\begin{array}{l}\text { MIC of teicoplanin } \geq \\
1.5 \mathrm{mg} / \mathrm{L}, \mathrm{n}(\%)\end{array}$ & $6(21.4)$ & $23(41.1)$ & 0.09 & $6(21.4)$ & $9(32.1)$ & 0.33 \\
\hline \multicolumn{7}{|l|}{ Comorbidities, n (\%) } \\
\hline $\begin{array}{l}\text { Coronary artery } \\
\text { disease }\end{array}$ & $12(42.9)$ & $5(8.9)$ & $\dot{0} 01$ & $12(42.9)$ & $3(10.7)$ & 0.03 \\
\hline Diabetes mellitus & $12(42.9)$ & $22(39.3)$ & 0.82 & $12(42.9)$ & $8(28.6)$ & 0.26 \\
\hline Hypertension & $18(64.3)$ & $25(44.6)$ & 0.11 & $18(64.3)$ & $11(39.3)$ & 0.08 \\
\hline Solid tumor & $10(35.7)$ & $9(16.1)$ & 0.06 & $14(50.0)$ & $4(14.3)$ & 0.08 \\
\hline $\begin{array}{l}\text { Hematology } \\
\text { malignancy }\end{array}$ & 0 & $1(1.8)$ & $\overrightarrow{0.99}$ & 0 & $1(3.6)$ & 0.61 \\
\hline Liver cirrhosis & $1(3.6)$ & $6(10.7)$ & 0.42 & $1(3.6)$ & $3(10.7)$ & 0.34 \\
\hline $\begin{array}{l}\text { Chronic obstructive } \\
\text { pulmonary disease }\end{array}$ & $3(10.7)$ & $4(7.1)$ & 0.68 & $3(10.7)$ & $2(71.4)$ & 0.66 \\
\hline IV drug abuser & 0 & $4(7.1)$ & 0.30 & 0 & $2(71.4)$ & 0.47 \\
\hline $\begin{array}{l}\text { Congestive heart } \\
\text { failure }\end{array}$ & $11(39.3)$ & $4(7.1)$ & $\dot{0} 01$ & $11(39.3)$ & $3(10.7)$ & 0.04 \\
\hline $\begin{array}{l}\text { End-stage renal } \\
\text { disease }\end{array}$ & $7(25.0)$ & $13(23.2)$ & $\overrightarrow{0.99}$ & $7(25.0)$ & $7(25.0)$ & $\overrightarrow{0} .99$ \\
\hline $\begin{array}{l}\text { Cerebral vascular } \\
\text { accident }\end{array}$ & $7(25.0)$ & $16(28.6)$ & 0.80 & $7(25.0)$ & $8(28.6)$ & 0.76 \\
\hline $\begin{array}{l}\text { Prosthetic device } \\
\text { implantation }\end{array}$ & $15(53.6)$ & $17(30.4)$ & 0.06 & 15 (53.6) & $7(25.0)$ & 0.05 \\
\hline
\end{tabular}




\begin{tabular}{|c|c|c|c|c|c|c|}
\hline Variables & $\begin{array}{l}\text { Initial } \\
\text { teicoplanin } \\
\text { treatment } \\
\mathrm{n}=\mathbf{2 8}\end{array}$ & $\begin{array}{l}\text { Initial ß- } \\
\text { lactam } \\
\text { treatment } \\
\mathrm{n}=56\end{array}$ & $P$ & $\begin{array}{l}\text { Initial } \\
\text { teicoplanin } \\
\text { treatment } \\
\mathbf{n = 2 8}\end{array}$ & $\begin{array}{l}\text { Initial ß- } \\
\text { lactam } \\
\text { treatment } \\
\mathbf{n = 2 8}\end{array}$ & $P *$ \\
\hline $\begin{array}{l}\text { Pittsburgh } \\
\text { bacteremia score } \geq \\
4^{\text {a }}\end{array}$ & $14(50.0)$ & $16(28.6)$ & 0.09 & $14(50.0)$ & $14(50.0)$ & NA \\
\hline \multicolumn{7}{|c|}{ Source of bacteremia ${ }^{b}, n(\%)$} \\
\hline $\begin{array}{l}\text { Catheter-related } \\
\text { bacteremia }\end{array}$ & $10(35.7)$ & $7(12.5)$ & 0.02 & $10(35.7)$ & $4(14.3)$ & 0.10 \\
\hline $\begin{array}{l}\text { Bone and joint } \\
\text { infection }\end{array}$ & $5(17.9)$ & $16(28.6)$ & 0.42 & $5(17.9)$ & $6(21.4)$ & 0.74 \\
\hline Infective endocarditis & 0 & $6(10.7)$ & 0.17 & 0 & $4(14.3)$ & 0.31 \\
\hline $\begin{array}{l}\text { Skin and soft } \\
\text { infection }\end{array}$ & $4(14.3)$ & $13(23.2)$ & 0.40 & $4(14.3)$ & $6(21.4)$ & 0.53 \\
\hline $\begin{array}{l}\text { Intra-abdominal } \\
\text { infection }\end{array}$ & 0 & $1(1.8)$ & $\overrightarrow{0.99}$ & 0 & $1(3.6)$ & 0.61 \\
\hline Urinary tract infection & 0 & $1(1.8)$ & $\overrightarrow{0} .99$ & 0 & $1(3.6)$ & 0.61 \\
\hline Pneumonia & $3(10.7)$ & $5(8.9)$ & $\overrightarrow{0.99}$ & $3(10.7)$ & $2(28.6)$ & 0.57 \\
\hline Primary bacteremia & $4(14.3)$ & $5(8.9)$ & 0.47 & $6(21.4)$ & $2(28.6)$ & 0.18 \\
\hline $\begin{array}{l}\text { Adequate infection } \\
\text { source control } \\
\text { rate }^{+} n / N\end{array}$ & 15/19 (78.9) & $\begin{array}{l}15 / 36 \\
(41.7)\end{array}$ & 0.01 & $15 / 19(78.9)$ & $\begin{array}{l}14 / 16 \\
(87.5)\end{array}$ & 0.67 \\
\hline \multicolumn{7}{|l|}{ Clinical outcome, n (\%) } \\
\hline $\begin{array}{l}\text { Short-term favorable } \\
\text { outcome }^{c}\end{array}$ & $11(39.3)$ & $34(60.7)$ & 0.10 & $11(39.3)$ & $11(39.3)$ & $\overrightarrow{0} .99$ \\
\hline Favorable outcome $^{d}$ & $24(85.7)$ & $43(76.8)$ & 0.40 & 24 (85.7) & $18(64.3)$ & 0.07 \\
\hline $\begin{array}{l}\text { 30-day overall } \\
\text { mortality }\end{array}$ & $4(14.3)$ & $13(23.2)$ & 0.40 & 4 (14.3) & $10(35.7)$ & 0.07 \\
\hline \multicolumn{7}{|c|}{$\begin{array}{l}\text { *Propensity score matching }(1: 1) \text { for age and Pittsburgh bacteremia score } \geq 4 \text { was performed for the } \\
\text { initial teicoplanin treatment group }(n=28) \text { versus the initial ß-lactam treatment group }(n=28) \text {. } \\
\text { Standardized mean difference (SMD) post-propensity score matching: age }(-2.78 \%) \text {, Pittsburgh } \\
\text { bacteremia score } \geq 4(0 \%)\end{array}$} \\
\hline \multicolumn{7}{|l|}{ NA: not applicable } \\
\hline
\end{tabular}




\begin{tabular}{|c|c|c|c|c|c|c|}
\hline Variables & $\begin{array}{l}\text { Initial } \\
\text { teicoplanin } \\
\text { treatment } \\
\mathrm{n}=\mathbf{2 8}\end{array}$ & $\begin{array}{l}\text { Initial B- } \\
\text { lactam } \\
\text { treatment } \\
\mathrm{n}=56\end{array}$ & $P$ & $\begin{array}{l}\text { Initial } \\
\text { teicoplanin } \\
\text { treatment } \\
\mathrm{n}=\mathbf{2 8}\end{array}$ & $\begin{array}{l}\text { Initial B- } \\
\text { lactam } \\
\text { treatment } \\
\mathbf{n = 2 8}\end{array}$ & $P *$ \\
\hline \multicolumn{7}{|c|}{$\begin{array}{l}\text { +Surgical intervention, drainage, central venous catheter removal, and urinary catheter removal were } \\
\text { performed for source control. Patients with pneumonia or primary bacteremia were excluded. } \mathrm{n} \text { : } \\
\text { adequate and timely removal or debridement of the source of bacteremia, } \mathrm{N} \text { : the source of bacteremia } \\
\text { needed to be removed or debrided. }\end{array}$} \\
\hline \multicolumn{7}{|c|}{${ }^{\mathrm{a}}$ At the time of blood sampling for culture. } \\
\hline \multicolumn{7}{|c|}{${ }^{\text {b Patients may have had more than one source of bacteremia. }}$} \\
\hline \multicolumn{7}{|c|}{${ }^{\mathrm{C}}$ Assessment on day 7 after starting the initial teicoplanin or ß-lactam antibiotic therapy. } \\
\hline
\end{tabular}

The risk factors for unfavorable clinical response at the completion of teicoplanin or $\beta$-lactam therapy in the unadjusted univariate analysis included higher disease severity (Pittsburgh bacteremia score $\geq 4$ $(94.1 \%$ versus $20.9 \%, p<0.01)$ and infective endocarditis as the source of bacteremia $(23.5 \%$ versus $3.0 \%$, $p=0.01$ ) (Table 2). After adjustments were made in the multivariate analysis, we observed that the Pittsburgh bacteremia score $\geq 4$ (odds ratio, $60.6 ; 95 \% \mathrm{Cl}, 7.4-496.8$ ) was independently associated with unfavorable outcome at the time of completion of teicoplanin or $\beta$-lactam therapy (Table 2). There was no significant evidence of lack of fit in any of the final models, as the $p$-values were $>0.05$ in the HosmerLemeshow goodness-of-fit tests. 
Table 2

Comparisons of demographic and clinical features between patients with methicillin-susceptible Staphylococcus aureus (MSSA) bacteremia with favorable and unfavorable final clinical responses

\section{Outcome at the end of the initial treatment Favorable outcome Unfavorable outcome $P$}

$$
n=67 \quad n=17
$$

\section{Demographics}

\begin{tabular}{|llll|}
\hline Male gender, $\mathbf{n}(\%)$ & $43(64.2)$ & $11(64.7)$ & $>0.99$ \\
\hline Age, mean (range), years & $68(59-77)$ & $72(48-85)$ & 0.82 \\
\hline MIC of teicoplanin $\geq 1.5 \mathrm{mg} / \mathrm{L}, \mathbf{n}(\%)$ & $22(32.8)$ & $7(41.2)$ & 0.57
\end{tabular}

Co-morbidities, $\mathrm{n}(\%)$

\begin{tabular}{|llll|}
\hline Coronary artery disease & $14(20.9)$ & $3(17.6)$ & $>0.99$ \\
\hline Diabetes mellitus & $30(44.8)$ & $4(23.5)$ & 0.17 \\
\hline Hypertension & $38(56.7)$ & $5(29.4)$ & 0.06 \\
\hline Solid tumor & $14(20.9)$ & $5(29.4)$ & 0.52 \\
\hline Hematology malignancy & $1(1.5)$ & 0 & $>0.99$ \\
\hline Liver cirrhosis & $4(6.0)$ & $3(17.6)$ & 0.14 \\
\hline Chronic obstructive pulmonary disease & $5(7.5)$ & $2(11.8)$ & 0.63 \\
\hline IV drug abuser & $2(3.0)$ & $2(11.8)$ & 0.18 \\
\hline Congestive heart failure & $13(19.4)$ & $2(11.8)$ & 0.73 \\
\hline End-stage renal disease & $17(25.4)$ & $3(17.6)$ & 0.75 \\
\hline Cerebral vascular accident & $21(31.3)$ & $2(11.8)$ & 0.14 \\
\hline Prosthetic device implantation & $27(40.3)$ & $5(29.4)$ & 0.58 \\
\hline Pittsburgh bacteremia score $\geq 4^{\mathrm{a}}$ & $14(20.9)$ & $16(94.1)$ & $<0.01^{\star}$ \\
\hline
\end{tabular}

+Surgical intervention, drainage, central venous catheter removal, and urinary catheter removal were performed for source control. Patients with pneumonia or primary bacteremia were excluded. $\mathrm{n}$ : adequate and timely removal or debridement of the source of bacteremia, $\mathrm{N}$ : the source of bacteremia needed to be removed or debrided.

${ }^{a}$ At the time of blood sampling for culture.

bPatients may have had more than one source of bacteremia.

*Multivariate analysis of the risk factors for an unfavorable clinical outcome in patients with MSSA bacteremia treated initially with teicoplanin or ß-lactam antibiotics showed that a Pittsburgh bacteremia score $\geq 4$ (odd ratio, 60.6 [95\% confidence interval, 7.4-496.8], $p<0.01$ ) was an independent risk factor for an unfavorable outcome. 


\begin{tabular}{|c|c|c|c|}
\hline Outcome at the end of the initial treatment & $\begin{array}{l}\text { Favorable outcome } \\
n=67\end{array}$ & $\begin{array}{l}\text { Unfavorable outcome } \\
n=17\end{array}$ & $P$ \\
\hline \multicolumn{4}{|l|}{ Source of bacteremiab ${ }^{b},(\%)$} \\
\hline Catheter-related bacteremia & $15(22.4)$ & $2(11.8)$ & 0.50 \\
\hline Bone and joint infection & $20(29.9)$ & $1(5.9)$ & 0.06 \\
\hline Infective endocarditis & $2(3.0)$ & $4(23.5)$ & 0.01 \\
\hline Skin and soft infection & $16(23.9)$ & $1(5.9)$ & 0.17 \\
\hline Intra-abdominal infection & 0 & $1(5.9)$ & 0.20 \\
\hline Urinary tract infection & $1(1.5)$ & 0 & $>0.99$ \\
\hline Pneumonia & $5(7.5)$ & $3(17.6)$ & 0.35 \\
\hline Primary bacteremia & $6(9.0)$ & $3(17.6)$ & 0.38 \\
\hline Adequate infection source control rate ${ }^{+} n / N$ & $28 / 51(54.9)$ & $2 / 4(50.0)$ & $>0.99$ \\
\hline \multicolumn{4}{|l|}{ Initial treatment group, n (\%) } \\
\hline Initial teicoplanin treatment group & $24(35.8)$ & $4(23.5)$ & 0.40 \\
\hline Initial ß-lactam treatment group & $43(64.2)$ & $13(76.5)$ & 0.40 \\
\hline \multicolumn{4}{|c|}{$\begin{array}{l}\text { +Surgical intervention, drainage, central venous catheter removal, and urinary catheter removal were } \\
\text { performed for source control. Patients with pneumonia or primary bacteremia were excluded. } \mathrm{n} \text { : } \\
\text { adequate and timely removal or debridement of the source of bacteremia, N: the source of bacteremia } \\
\text { needed to be removed or debrided. }\end{array}$} \\
\hline \multicolumn{4}{|l|}{${ }^{\mathrm{a}}$ At the time of blood sampling for culture. } \\
\hline \multicolumn{4}{|c|}{ bPatients may have had more than one source of bacteremia. } \\
\hline \multicolumn{4}{|c|}{$\begin{array}{l}\text { *Multivariate analysis of the risk factors for an unfavorable clinical outcome in patients with MSSA } \\
\text { bacteremia treated initially with teicoplanin or } \beta \text {-lactam antibiotics showed that a Pittsburgh } \\
\text { bacteremia score } \geq 4 \text { (odd ratio, } 60.6 \text { [ }[95 \% \text { confidence interval, } 7.4-496.8], p<0.01 \text { ) was an } \\
\text { independent risk factor for an unfavorable outcome. }\end{array}$} \\
\hline
\end{tabular}

All patients included in the study were divided into the initial teicoplanin treatment $(n=28)$ and $\beta$-lactam treatment $(n=28)$ groups after $1: 1$ propensity score matching with Pittsburgh bacteremia score $\geq 4$ (the independent risk factor for unfavorable outcome) and age. After matching, there were no statistically significant differences in terms of sex, length of hospital stay, and the proportion of $S$. aureus strains with teicoplanin MICs $\geq 1.5 \mathrm{mg} / \mathrm{L}$ between the groups. The incidence of coronary artery disease and congestive heart failure was higher $(42.9 \%$ versus $10.7 \%, p=0.03$ and $39.3 \%$ versus $10.7 \%, p=0.04$, respectively) among patients receiving initial teicoplanin treatment than in those receiving $\beta$-lactam 
treatment even after propensity score matching. No statistically significant differences were observed in the source of bacteremia and the rate of adequate infection source control between the two groups after matching. We did not find statistically significant differences in short-term favorable outcome, favorable outcome at the time of completion of teicoplanin or $\beta$-lactam therapy, and 30-day overall mortality rate between the groups after propensity score matching.

Among the patients in the initial teicoplanin treatment group, 21 (75.0\%) switched to $\beta$-lactam treatment and three $(14.3 \%)$ died within 30 days of hospitalization after the onset of MSSA bacteremia. Altogether, $71.5 \%(15 / 21)$ of the patients switched to $\beta$-lactam treatment within 4 days after the onset of bacteremia, which was consistent with the time of availability of final susceptibility test results (Supplementary Figure). On the other hand, the 30 -day mortality rate was $14.3 \%$ in patients with initial teicoplanin treatment without a switch to $\beta$-lactam treatment for MSSA bacteremia (Table 1). The clinical data of seven patients with MSSA bacteremia who received continuous teicoplanin treatment are shown in Supplementary Table. The MIC distributions of teicoplanin in the MSSA strains isolated from patients in the initial teicoplanin treatment group $(n=28)$ and the $\beta$-lactam treatment group $(n=56)$ are shown in Fig. 2. We observed that $78.6 \%(22 / 28)$ of the MSSA strains in the initial teicoplanin treatment group exhibited teicoplanin MICs $<1.5 \mathrm{mg} / \mathrm{L}$, while $60.7 \%(34 / 56)$ of the MSSA strains in the initial $\beta$-lactam treatment group exhibited teicoplanin MICs $<1.5 \mathrm{mg} / \mathrm{L}$.

The Kaplan-Meier curves for 30-day survival in patients with MSSA bacteremia are presented in Fig. 3. The cases were grouped according to the initial treatment (teicoplanin or $\beta$-lactam) before propensity score matching (Fig. 3(i)) and were further stratified as the initial teicoplanin treatment group or the $\beta$ lactam treatment group after propensity score matching (Fig. 3(ii)). The 30-day survival was not significantly different between the two groups before propensity score matching (hazard ratio, 1.84; 95\%

$\mathrm{Cl}, 0.60-5.64, \mathrm{p}=0.29$ ) as well as after propensity score matching (hazard ratio, $3.12 ; 95 \% \mathrm{Cl}, 0.98-9.99$, $p=0.06)$.

\section{Discussion}

Several studies regarding initial vancomycin treatment for MSSA bacteremia have revealed unfavorable outcomes [7-13]. Lodise et al reported that 72 patients with MSSA-related infective endocarditis who were initially treated with vancomycin and subsequently switched to a $\beta$-lactam antibiotic in a median duration of 3 days had a four-fold increase in the mortality compared to those who were initially treated with a $\beta$-lactam antibiotic (9/22 [40.9\%] versus $5 / 44$ [11.4\%]) [6]. This finding suggests that the timing initiation of appropriate empirical therapy is critical for the outcomes, since even the patients who switched to $\beta$-lactam therapy after the availability of susceptibility results showed outcomes inferior to those treated with a $\beta$-lactam antibiotic initially. To the best of our knowledge, our study, which consisted of a cohort of 84 patients with MSSA bacteremia, is the first study to examine the clinical outcomes in patients who received teicoplanin or $\beta$-lactam treatment. In our study, patients in the initial teicoplanin treatment group did not seem to exhibit poorer outcome than those in the $\beta$-lactam treatment group. Even after propensity score matching for disease severity, patients in the initial teicoplanin treatment group 
exhibited similar clinical outcomes when compared with patients in the $\beta$-lactam treatment group. No empirical regimen involving a switch from teicoplanin to $\beta$-lactam was found to be superior or inferior to the other even when patients switched to $\beta$-lactam treatment after the availability of culture results. This relationship persisted in the logistic regression analysis controlling for clinical characteristics. This finding may be related to the timing of the switch to $\beta$-lactam treatment in our cohort, which occurred less than 4 days after the onset of infection in 53.6\% (21/28) (Supplementary Figure). Another issue regarding the relationship of treatment outcomes with teicoplanin MICs has been discussed by Chang et al [22]. They suggested that MRSA bacteremia with a teicoplanin MIC $\geq 1.5 \mathrm{mg} / \mathrm{L}$ was a predictive factor for unfavorable outcomes in patients receiving teicoplanin treatment [22]. In the present study, $78.6 \%(22 / 28)$ of the patients in the initial teicoplanin treatment group with MSSA bacteremia had teicoplanin MICs < $1.5 \mathrm{mg} / \mathrm{L}$ (Fig. 2). This result may partially explain the absence of statistically significant unfavorable clinical outcomes in the initial teicoplanin treatment group. Moreover, our recent study indicated that patients with MRSA bacteremia who received teicoplanin treatment with a maintenance dose of $6 \mathrm{mg} / \mathrm{kg}$ every 12 hours exhibited favorable outcomes irrespective of the teicoplanin MIC [31]. Further studies with larger sample sizes may be needed to confirm our findings.

A previous study indicated that in vitro bactericidal activity of vancomycin is slower than that of nafcillin, with more frequent failures in animal models [32]. In our present study, patients with MSSA bacteremia in the initial $\beta$-lactam treatment group exhibited a higher rate of favorable short-term outcomes than those in the initial teicoplanin treatment group (60.7\% versus $39.3 \%)$. However, the difference was not statistically significant, which might be due to our small sample size. Some studies conducted in special patient groups such as hemodialysis-dependent and bloodstream-related pneumonia patients with MSSA bacteremia demonstrated high rates of treatment failure and mortality among patients receiving vancomycin treatment $[10,11]$. Among patients with MSSA bacteremia, the 30 -day mortality or unfavorable outcomes were not significantly higher in patients with infection due to pneumonia and in hemodialysis-dependent patients when compared with those having other sources of infection. This result seems to be inconsistent with the findings of Stryjewski et al [10]. Although the lung penetration of teicoplanin may be lower than that of vancomycin [33,34], a higher dose of teicoplanin would be more effective [31], particularly in patients with bacteremic pneumonia. Other reasons may be related to the small sample size of this patient population in our study and the fact that only one patient was infected by a bacterial strain with teicoplanin MIC $\geq 1.5 \mathrm{mg} / \mathrm{L}$.

The present study still has some limitations. The retrospective study design has inherent limitations due to potential confounding and selection bias. Prospective randomized controlled trials are needed to validate our findings. A small proportion of patients were diagnosed with endocarditis in the present study. Variations in the source of MSSA bacteremia and the small number of patients with endocarditis precludes us from making comparisons the outcomes of endocarditis with those of other more common sources of MSSA bacteremia. We did not analyze serum teicoplanin levels in the present study. However, all patients received teicoplanin treatment with a maintenance dose of $6 \mathrm{mg} / \mathrm{kg}$ every 12 hours. Our previous experience has shown that this dosage will produce favorable outcomes in patients with MRSA bacteremia irrespective of the teicoplanin MIC [31]. 


\section{Conclusions}

The results of the present study indicated that there was no significant difference in the clinical outcomes between patients with MSSA bacteremia receiving initial teicoplanin treatment (maintenance dose of $6 \mathrm{mg} / \mathrm{kg}$ every 12 hours) and those receiving $\beta$-lactam treatment before the availability of susceptibility report. Pittsburgh bacteremia score $\geq 4$ was an independent risk factor for mortality.

\section{Abbreviations}

MSSA: methicillin-sensitive Staphylococcus aureus; MRSA: methicillin-resistant Staphylococcus aureus; MIC: minimum inhibitory concentration; $\mathrm{Cl}$ : confidence interval

\section{Declarations}

\section{Ethics approval and consent to participate}

The study was approved by the Institutional Review Board of Chang Gung Memorial Hospital (No. 201601482B0).

\section{Funding}

This work was supported by grants from Chang Gung Memorial Hospital, Taiwan (CMRPG 8J0391). They had no role in the design of the study and collection, analysis, and interpretation of data and in writing the manuscript.

\section{Availability of data and materials}

The datasets used and/or analysed during the current study are available from the corresponding author on reasonable request.

\section{Consent for publication}

Not Applicable.

\section{Competing interests}

The authors declare no conflicts of interest.

\section{Authors' contributions}

Ching-Yen Tsai: the first author with clinical data collection and manuscripts writing.

Chen-Hsiang Lee: corresponding author

I-Ling Chen: assistant and data statistical analysis 


\section{Acknowledgments}

We would like to thank the Biostatistics Center, Kaohsiung Chang Gung Memorial Hospital for the statistical analyses. We also thank Dr. Chien-Ching Hung at the Department of Internal Medicine, National Taiwan University Hospital, for his critical review of this manuscript.

\section{References}

1. Valles J, Rello J, Ochagavia A, Garnacho J, Alcala MA. Community-acquired bloodstream infection in critically ill adult patients: impact of shock and inappropriate antibiotic therapy on survival. 2003; 123(5):1615-24.

2. Leibovivi L, Shraga I, Drucker M, Konigsberger H, Samra Z, Pitlik SD. The benefit of appropriate empirical antibiotic treatment in patients with bloodstream infection. J Intern Med. 1998; 244(5):279-86.

3. Kollef MH, Ward S, Sherman G, et al. Inadequate treatment of nosocomial infections is associated with certain empiric antibiotic choices. Crit Care Med. 2000; 28(10):3456-64.

4. Ibrahim EH, Sherman G Ward S, Fraser VJ, Kollef MH. The influence of inadequate antimicrobial treatment of blood-stream infections on patient outcomes in the ICU setting. Chest. 2000; 118(1):146-55.

5. Roghmann MC. Predicting methicillin resistance and the effect of inadequate empiric therapy on survival in patients with Staphylococcus aureus bacteremia. Arch Intern Med. 2000; 160(7):1001-4.

6. Lodise TP, McKinnon PS, Swiderski L, Rybak MJ. Outcomes analysis of delayed antibiotic treatment for hospital-acquired Staphylococcus aureus bacteremia. Clin Infect Dis. 2003; 36(11):1418-23.

7. Johnson LB, Almoujahed MO, Ilg K, Maolood L, Khatib R. Staphylococcus aureus bacteremia: compliance with standard treatment, long-term outcome and predictors of relapse. Scand J Infect Dis. 2003; 35(11-12):782-9.

8. Khatib R, Saeed S, Sharma M, Riederer K, Fakih MG, Johnson LB. Impact of initial antibiotic choice and delayed appropriate treatment on the outcome of Staphylococcus aureus bacteremia. Eur $\mathrm{J}$ Clin Microbiol Infect Dis. 2006; 25(3):181-5.

9. Chang FY, MacDonald BB, Peacock JE Jr, et al. A prospective multicenter study of Staphylococcus aureus bacteremia: incidence of endocarditis, risk factors for mortality, and clinical impact of methicillin resistance. Medicine. 2003; 82(5):333-9.

10. Stryjewski ME, Szczech LA, Benjamin DK Jr, et al. Use of vancomycin or first-generation cephalosporins for the treatment of hemodialysis-dependent patients with methicillin-susceptible Staphylococcus aureus bacteremia. Clin Infect Dis. 2007; 44(2):190-6.

11. González C, Rubio M, Romero-Vivas J, González M, Picazo JJ. Bacteremic pneumonia due to Staphylococcus aureus: A comparison of disease caused by methicillin-resistant and methicillinsusceptible organisms. Clin Infect Dis. 1999; 29(5):1171-7. 
12. Lodise TP Jr, McKinnon PS, Levine DP, Rybak MJ. Impact of empirical-therapy selection on outcomes of intravenous drug users with infective endocarditis caused by methicillin-susceptible Staphylococcus aureus. Antimicrob Agents Chemother. 2007; 51(10):3731-3.

13. McConeghy KW, Bleasdale SC, Rodvold KA. The empirical combination of vancomycin and a $\beta$ lactam for Staphylococcal bacteremia. Clin Infect Dis. 2013; 57(12):1760-5.

14. Svetitsky S, LeiboviciL, Paul M. Comparative efficacy and safety of vancomycin versus teicoplanin: systematic review and meta-analysis. Antimicrobe Agents Chemother. 2009; 53(10):4069-79.

15. BabuIN, Pasko M. Teicoplanin: a new glycopeptide antibiotic complex. Drug Intell Clin Pharm. 1988; 22(3):218-26.

16. Cavalcanti AB, Goncalves AD, Almeida CS, Bugano DD, Silva E. Teicoplanin versus vancomycin for proven or suspected Infection. Cochrane Database Syst Rev. 2010; 16;(6):CD007022.

17. Hiroko $\mathrm{KH}$, Takashi $\mathrm{N}$, Koichi $\mathrm{O}$, et al. Comparative efficacy and safety of vancomycin versus teicoplanin in febrile neutropenic patients receiving hematopoietic stem cell transplantation. J Clin Pharm Ther. 2019; 44(6):888-94.

18. Tsai $\mathrm{CY}$, Lee $\mathrm{CH}$, Chen IL. Daptomycin versus teicoplanin for bloodstream infection due to methicillinresistant Staphylococcus aureus with a high teicoplanin minimal inhibitory concentration $\geq 1.5$ mg/L: a propensity score-based analysis. Infect Drug Resist. 2018; 11:2011-20.

19. Tsai CY, Lee CH, Chie CC, Chen IL. Impact of teicoplanin maintenance dose and MIC values on the clinical outcomes of patients treated for methicillin-resistant Staphylococcus aureus Infect Drug Resist. 2018; 11:1205-17.

20. Zhou L, Gao Y, Cao W, Liu J, Guan H, Zhang H, et al. Retrospective analysis of relationships among the dose regimen, through concentration, efficacy, and safety of teicoplanin in Chinese patients with moderate-severe Gram-positive infection. Infect Drug Resist. 2018; 11:29-36.

21. Gilbert ND, Moellering Jr. RC, Eliopoulos GM, Chambers HF, Saag MS. Selected pharmacologic features of antimicrobial agents. The Sanford Guide to Antimicrobial Therapy. 2009; 78-82.

22. Chang HJ, Hsu PC, Yang CC, et al. Influence of teicoplanin MICs on treatment outcomes among patients with teicoplanin-treated methicillin-resistant Staphylococcus aureus bacteraemia: A hospital-based retrospective study. J Antimicrob Chemother. 2012; 67(3):736-41.

23. Chen IL, Lee CH, Su LH, Chang SJ, Liu JW. Antibiotic consumption and healthcare-associated infections caused by multidrug-resistant gram-negative bacilli at a large medical center in Taiwan from 2002 to 2009: Implicating the importance of antibiotic stewardship. PLoS One. 2013; 8(5):e65621.

24. Clinical and Laboratory Standards Institute. Performance standards for antimicrobial susceptibility testing; 25th informational supplement, CLSI document M100-S25. CLSI, Wayne, PA. 2015.

25. Chow JW, Yu VL. Combination antibiotic therapy versus monotherapy for gram-negative bacteremia: a commentary. Int J Antimicrob Agents. 1999;11(1):7-12

26. Brun-Buisson C, Abrouk F, Legrand P, Huet Y, Larabi S, Rapin M. Diagnosis of central venous catheterrelated sepsis. Critical level of quantitative tip cultures. Arch Intern Med. 1987; 147(5):873-7. 
27. Garner JS, Jarvis WR, Emori TG, Horan TC, Hughes JM. CDC definitions for nosocomial infections. Am J Infect Control. 1988; 16(3):128-40.

28. El-Ahdab F, Benjamin DK Jr, Wang A, et al. Risk of endocarditis among patients with prosthetic valves and Staphylococcus aureus bacteraemia. Am J Med. 2005; 118(3):225-9.

29. Christou NV, Turgeon P, Wassef R, Rotstein O, Bohnen J, Potvin M. Management of intra-abdominal infections. The case for intra-operative cultures and comprehensive broad-spectrum antibiotic coverage. The Canadian Intra-abdominal Infection Study Group. Arch Surg. 1996; 131(11):1193201.

30. Liu C, Bayer A, Cosgrove SE, et al. Clinical practice guidelines by the Infectious Diseases Society of America for the treatment of methicillin-resistant Staphylococcus aureus infections in adults and children: executive summary. Clin Infect Dis. 2011; 52(3):285-92.

31. Lee CH, Tsai CY, Li CC, Chien CC, Liu JW. Teicoplanin therapy for MRSA bacteraemia: a retrospective study emphasizing the importance of maintenance dosing in improving clinical outcomes. J Antimicrob 2015; 70(1):257-63.

32. Cantoni L, Glauser MP, Bille J. Comparative efficacy of daptomycin, vancomycin and cloxacillin for the treatment of Staphylococcus aureus endocarditis in rats and role of test conditions in this determination. Antimicrob Agents Chemother. 1990; 34(12):2348-53.

33. Welte T, Pletz MW. Antimicrobial treatment of nosocomial methicillin-resistant Staphylococcus aureus (MRSA) pneumonia: current and future options. Int J Antimicrob Agents. 2010; 36(5):391400.

34. Mimoz O, Rolland D, Adoun M, et al. Steady-state trough serum and epithelial lining fluid concentrations of teicoplanin $12 \mathrm{mg} / \mathrm{kg}$ per day in patients with ventilator-associated pneumonia. Intensive Care Med. 2006; 32(5):775-9.

\section{Figures}


246 Staphylococcus aureus bacteremia patients were hospitalized between September 2012 and September 2014

\section{$155(63 \%)$ MRSA bacteremia patients were excluded}

91 MSSA bacteremia patients

- 2 patients : no any antibiotics treatment

- 2 patients : initial vancomycin treatment

- 3 patients : age $<18$ years old

84 MSSA bacteremia patients were enrolled

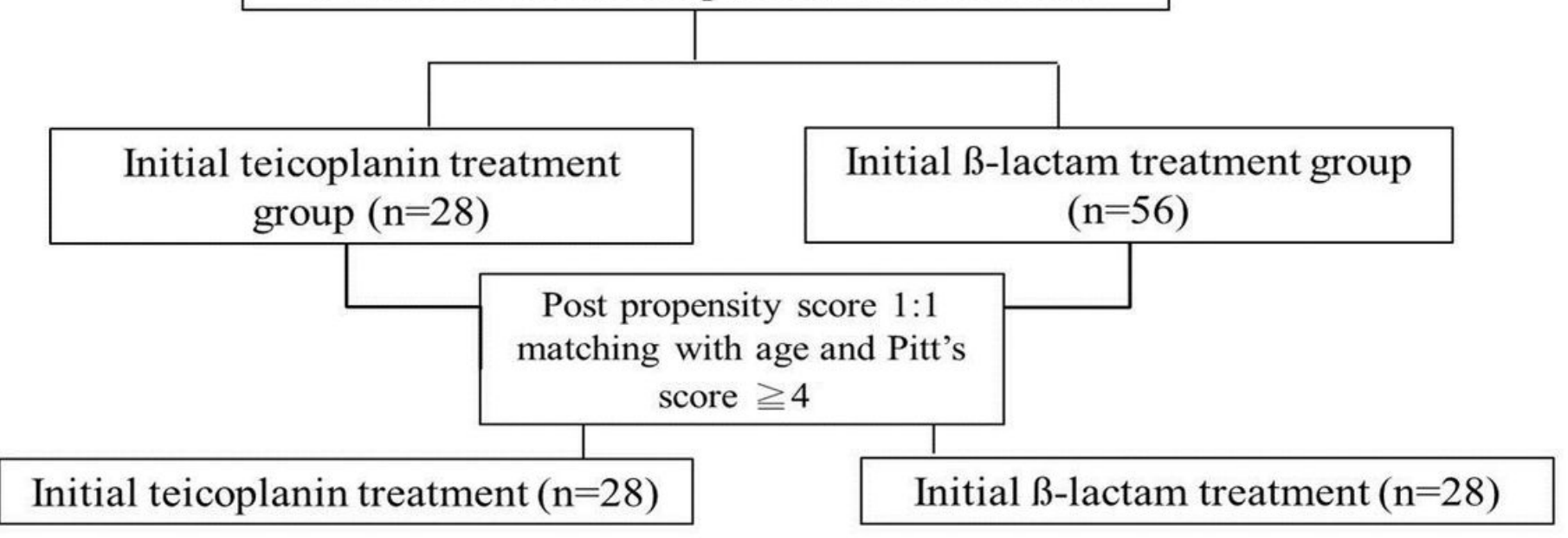

\section{Figure 1}

Flow chart of the inclusion and categorization of patients with methicillin-susceptible Staphylococcus aureus (MSSA) bacteremia receiving initial teicoplanin or ß-lactam treatment. 


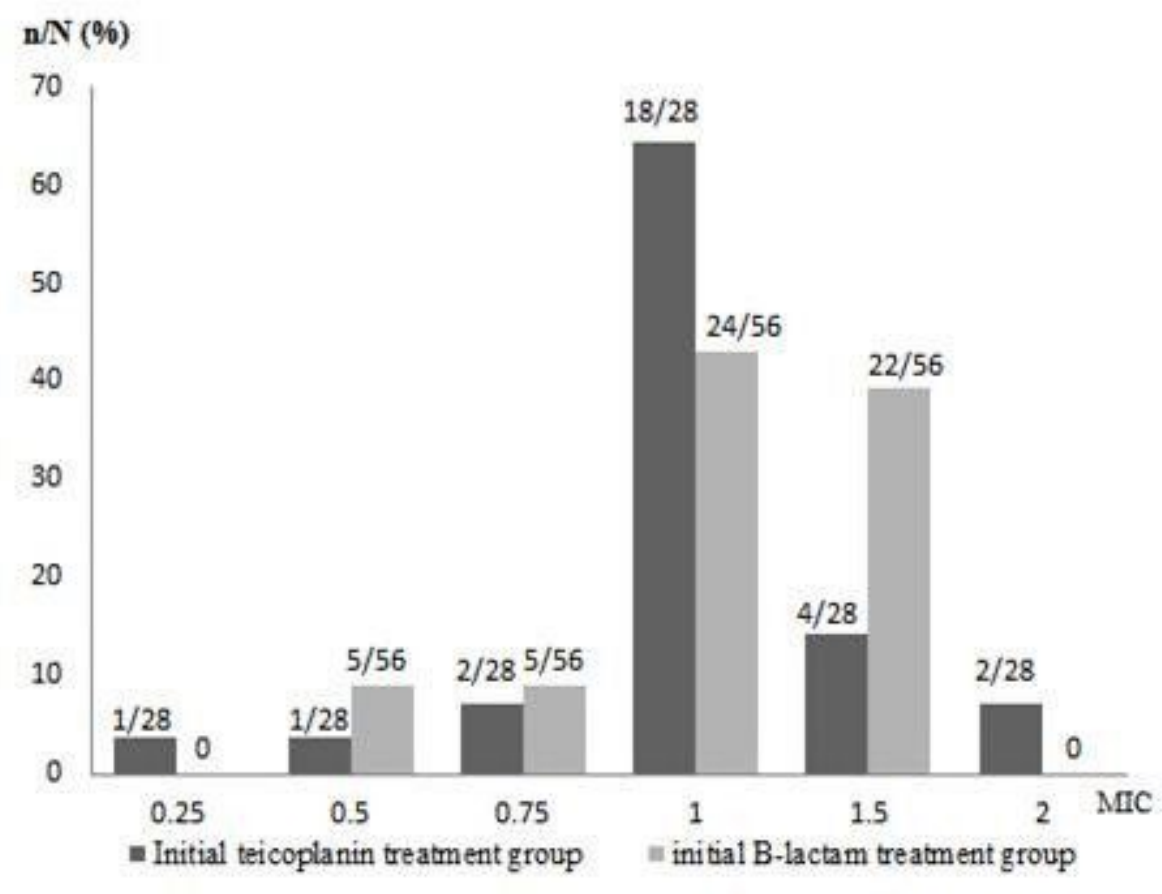

\section{Figure 2}

Distribution of minimum inhibitory concentration (MIC) of teicoplanin in the initial teicoplanin treatment $(n=28)$ and $ß$-lactam treatment $(n=56)$ groups. In total, $78.6 \%(22 / 28)$ of patients in the initial teicoplanin treatment group with methicillin-susceptible Staphylococcus aureus (MSSA) bacteremia had teicoplanin MICs $<1.5 \mathrm{mg} / \mathrm{L}$ and $60.7 \%$ (34/56) of patients in the initial ß-lactam treatment group with MSSA bacteremia had teicoplanin MICs $<1.5 \mathrm{mg} / \mathrm{L}$. 

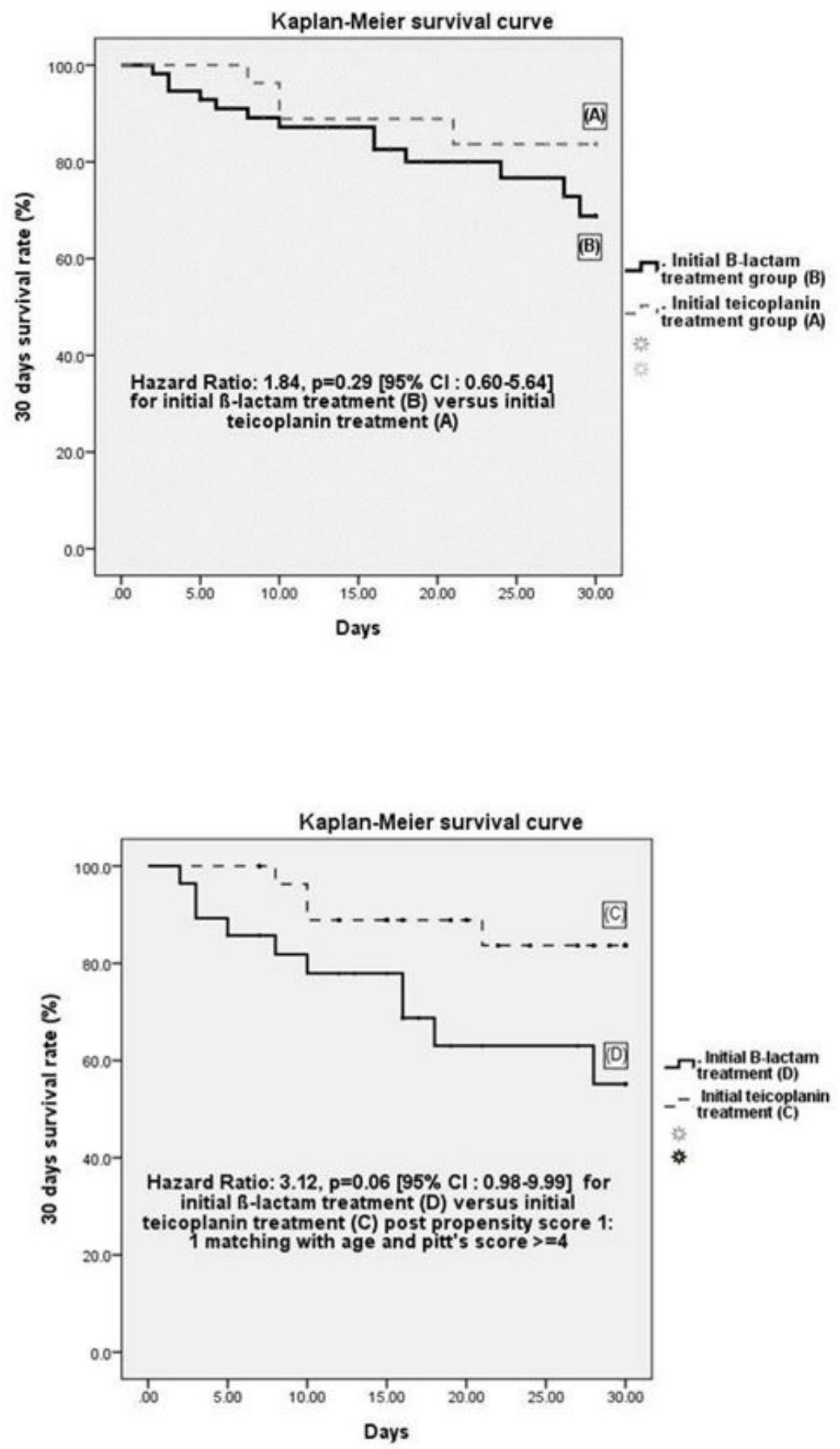

\section{Figure 3}

Kaplan-Meier curve for 30-day survival of patients with methicillin-susceptible Staphylococcus aureus (MSSA) bacteremia treated initially with teicoplanin or ß-lactam antibiotics. (i) Cases are grouped according to the initial treatment: teicoplanin (A) or $B$-lactam antibiotics (B). (ii) Cases are grouped according to the initial treatment: teicoplanin (C) or ß-lactam antibiotics (D) after 1:1 propensity score matching for age and Pittsburgh bacteremia score $\geq 4$. 


\section{Supplementary Files}

This is a list of supplementary files associated with this preprint. Click to download.

- SupplementaryTable.docx

- FigS1.jpg 